\title{
PROCESS AND SYSTEMS A population-based model of care for people with inflammatory bowel disease - patient-reported outcomes
}

\author{
Authors: Antony Aziz, ${ }^{A}$ Rebecca Reynolds ${ }^{B}$ and Azhar Ansari ${ }^{C}$
}

The NHS was not designed to provide ongoing support for people with long-term conditions. Conventional outpatient care relies on a diary-based appointment system, with regular follow-up offered to patients with a chronic disorder, not always tailored to clinical need. In contrast, at East Surrey Hospital, open access to the inflammatory bowel disease (IBD) service through telephone, email and a web-based portal known as Patients Know Best is offered to all people with IBD, putting them at the centre of the care pathway. This guides and directs those with the greatest clinical need to the clinician with the most appropriate clinical expertise to provide high quality consistent care. Over a 3 month period in 2015, the service avoided 20 hospital admissions, 34 emergency department attendances and 110 outpatient appointments. There is a demonstrable improvement in perception of IBD control and in the patient activation measure, with $66 \%$ of those who have used the open access service demonstrating medium to high levels of activation, compared with $11 \%$ in those new to the service.

KEYWORDS: Person-centred care, IBD, system change, new models of care

\section{Introduction}

The birth of our NHS in 1948 was a great moment for healthcare in the UK, promising good healthcare for all that is free at the point of care. Since its inception, the NHS principles and values have revolved around putting patients at the heart of everything it does. The NHS constitution emphasises patients' rights to be involved in all aspects of their healthcare and the NHS Five Year Forward View published in 2014 highlights the need for supporting and empowering patients to manage their own health better. ${ }^{1,2}$

The NHS was not designed to provide ongoing support for people with long-term conditions who continue to be disproportionate

Authors: A gastroenterology registrar and Darzi fellow, East Surrey Hospital, Redhill, UK; ${ }^{\text {B }}$ gastroenterology registrar, East Surrey Hospital, Redhill, UK; ' Cgastroenterology consultant, East Surrey Hospital, Redhill, UK users of the health system. ${ }^{3}$ Approximately 15 million people in England have a long-term condition and they now account for about $50 \%$ of all general practitioner (GP) appointments, $64 \%$ of all outpatient appointments and over $70 \%$ of all inpatient bed days. ${ }^{4}$ Treatment and care for people with long-term conditions is estimated to take up around $£ 7$ in every $£ 10$ of total health and social care expenditure in the UK. ${ }^{4}$ People with a long-term condition will most likely spend less than $1 \%$ of their time in contact with healthcare professionals and manage on their own with the support of their families and carers the rest of the time. ${ }^{2}$

Conventional outpatient care still relies on a diary-based appointment system, with regular follow-up offered to patients with chronic disorder. With the increasing burden of chronic disease and the ongoing lack of trained healthcare professionals, many outpatient services are faced with long waiting times as demand exceeds capacity. Furthermore, people with long-term conditions experience unpredicted flare-ups that either require an additional appointment, or a visit to their GP or emergency department if the outpatient service is not able to accommodate them. Delay in intervention is associated with poor clinical outcomes, increased hospital admissions and increased cost to the citizen and the healthcare economy. ${ }^{5}$

The chronic care model described by Wagner in 1998 puts emphasis on supporting self-management, reorganising team function and clinical practice systems, and improving information systems in order to improve outcomes. ${ }^{6}$ We demonstrate how we have created a new model of care that revolutionises chronic disorder management through a population-based model in inflammatory bowel disease.

\section{Case study}

East Surrey Hospital (ESH) is a large district general hospital (DGH) in the south east of England that serves a population of 535,000. With inflammatory bowel disease (IBD) affecting $0.5-1 \%$, this equates to between 2,700 and 5,400 IBD patients. The IBD service at ESH already looks after more than 3000 patients. According to national British Society of Gastroenterology (BSG) guidelines, the ESH population should be supported by four whole time equivalent (WTE) IBD consultants and three WTE IBD clinical nurse specialists (CNS). ${ }^{7}$ Since 2010 , the service has been run with one and half WTE IBD consultants and one WTE IBD CNS. This mismatch in manpower, resource and demand on 
services resulted in long waiting times for conventional outpatient care and adverse outcomes for IBD patients unable to access services when required and often relying on admission to hospital through the emergency department for treatment. In 2012, the gastroenterology department at ESH estimated it had 300 IBD patients known to its service for the national IBD audit. This is less than $10 \%$ of the currently known IBD population in the hospital's catchment. This difference between apparent demand and true demand is likely to be due to poor data quality and the absence of a patient database, but may be in part attributed to a mismatch between demand and capacity where activity is largely driven by capacity in a constrained diary-based outpatient service that does not match clinical need with resource.

\section{The current service}

True demand on a service can only be realised by providing open access and removing the protective shield surrounding the silo-based healthcare system reliant on diarised appointments. Giving patients direct access to their specialist using a technologydriven service they own, not limited to opening hours, location or availability of staff uncovers previously unmet needs. To maintain this service requires a different structure of support with administrators and CNSs playing a key role in responding to patients' requests and offering appropriate advice and escalation.

In 2014, open access to the IBD service through telephone and email support was offered to all patients with IBD, putting them at the centre of the care pathway and enabling them to drive the change. This led to the introduction of a web-based patient management portal called Patients Know Best (PKB). This nonface-to-face (NFTF) delivery of service empowered patients to become authors of their own health data (see Box 1). They record their own symptoms and communicate these to the IBD team remotely, enabling access to timely advice and clinical review in the context of a flare, and escalation to disease-modifying therapy tailored to the individual whilst reassuring those that are stable without the need for a face-to-face review.

This intervention guides and directs patients with the greatest clinical need to the clinician with the most appropriate clinical expertise to provide high quality consistent care. All NFTF interactions are vetted by an IBD CNS with administrator support, increasing their exposure to the local IBD population, allowing them to better understand local health needs while up-skilling them through consultant supervision. Consultant face-to-face time in clinic is released from routine follow-up of stable patients and

Box 1. Key features of the Patients Know Best webbased portal

Access to personalised healthcare record including: diagnosis, current and past medications with previous adverse reactions if applicable and links to patient information resources

Access to investigation results through integration with the trust IT reporting system

Disease activity assessment tools and symptom tracker: stool frequency, blood in stools, abdominal pain, fatigue, urgency

Improved communication with the IBD team including secure messaging with the ability to add file attachments (documents or photographs) diverted to those with complex disease or who are experiencing a flare.

The current payment-by-results commissioning structure leads to poor remuneration for chronic disease follow-up, where new appointments are given priority. With the current funding model, there is a drive to recruit more consultants in order to provide greater outpatient capacity. However, we have demonstrated that different resource distribution and process structures enable the true clinical need to be met with fewer staff and at lower cost. Therefore, commissioning ought to support improved outcomes and not higher activity.

To provide resilience and sustainability to the NFTF IBD service, early discussions took place between ESH and local clinical commissioning groups (CCGs). ESH continued to fund the PKB licence on a year-by-year pilot basis as it recognised the quality and safety improvements seen. Further commissioning is now being supported through block contracting with CCGs in order to provide future resilience to the service, until there is a cultural shift in healthcare commissioning in favour of improved outcomes.

Treatment of IBD, like most chronic disorders, has evolved greatly over the past two decades and early, aggressive treatment with immunosuppression is recommended to reduce the adverse effects of the inflammatory process. ${ }^{8}$ Azathioprine is commonly used as first line oral therapy and requires close monitoring for adverse reactions. If tolerated, its use is associated with reduced intestinal inflammation, reduced risk of dysplasia and fewer surgeries, hospitalisation and steroid use over many years. ${ }^{9,10}$ However, up to $40 \%$ of patients will stop azathioprine due to dose-related side effects and drug hypersensitivity reactions. ${ }^{11}$ The user experience is commonly poor due to slow onset of action coupled with a lack of open access to care and resulting delay in reporting side effects. Treatment is then escalated to costly monoclonal antibody therapy in an attempt to induce remission. Its rapid onset of action, higher rate of positive response and lower side effect rate make it popular amongst clinicians. ${ }^{11}$ However, it requires infusion or injection and often loses effect over time. Therefore, the optimisation of azathioprine is still preferable.

At ESH, azathioprine use has been enhanced to increase its effectiveness from the outset. Its use at a low dose when combined with allopurinol improves efficacy, reduces side effects and is better tolerated, thus reducing the need for clinical intervention and escalation to monoclonal therapy. It can be managed entirely NFTF. ${ }^{12}$

Current practice sees approximately $10-15 \%$ of IBD patients on monoclonal therapy. ${ }^{13}$ This predicts $300-450$ patients at ESH. As of June 2018 , the actual figure was 140 without concern for undertreatment due to the use of low-dose azathioprine allopurinol co-therapy coupled with early and open access to the service. Monitoring is done as per national guidance with annual review and is also offered NFTF where appropriate according to patient preference (Fig 1).

\section{Service evaluation}

\section{Methods}

In 2015, data was extracted from all telephone, email and PKB interactions over a 3-month period. Each interaction was categorised to determine whether it was regarding diagnosis, flare management, medication monitoring, or any other query. A team consisting of the IBD consultant, CNS and administrator 


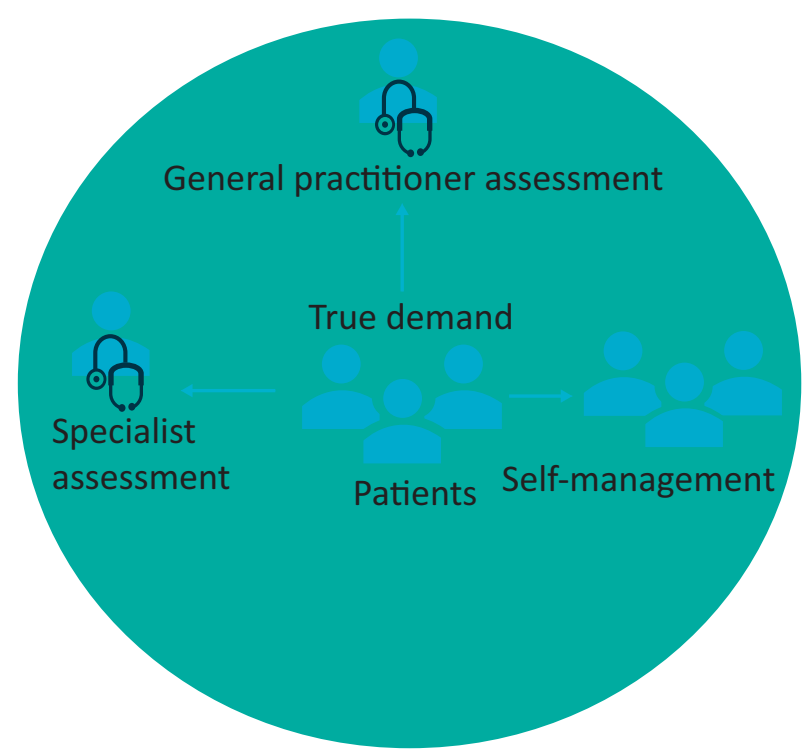

Fig 1. Technology-enhanced open access healthcare service. The new model for outpatient care. The patient is at the centre of the model and has open access to services via telephone, email and Patients Know Best with the majority engaging in supported self-management via the web-based portal.

determined whether the outcome of each interaction resulted in an avoided hospital admission, emergency department attendance or outpatient appointment.

In 2018, the service underwent an evaluation in terms of patient self-management, patient-reported outcome measures and clinical outcomes. Data was collected from 35 patients in a prospective cohort using two questionnaires taken at 0 and 4 months post introduction to NFTF services. The first questionnaire is the Patient Activation Measure ${ }^{\circledR}\left(\right.$ PAM $\left.^{\circledR}\right)$ survey, which asks 13 questions that focus on the knowledge, skills and confidence that individuals have to manage their health. It provides a score on a 100 point scale that correlates with clinical outcomes which is further categorised into a four level tier scale. ${ }^{14}$ The four levels of activation are shown below as 'Low' (Levels 1 \& 2), 'Moderate' (Level 3), and 'High' (Level 4). The second questionnaire is the IBD-Control questionnaire, a rapid, reliable, valid and sensitive instrument co-produced with patients based on focus groups and interviews for measuring overall disease control from the patient's perspective. ${ }^{15}$ By combining the two questionnaires, the hypothesis is that, as part of a wider picture, the NFTF IBD service provides timely care, improves self-efficacy and has a positive impact on patient-reported outcomes. This data was compared with the same data collected from 35 patients in a retrospective cohort with over 12 months experience of using the ESH IBD NFTF service. All patients were recruited to the study through the IBD consultant clinic over a 2 month period. Secondary data is being gathered on the number of outpatient appointments, emergency department attendances, inpatient admissions and courses of steroids prescribed for each cohort over one year, but this data is not yet available.

\section{Results}

In 2015, there were 4,358 NFTF interactions in total. Over the 3-month period, the service avoided 20 hospital admissions, 34 emergency department attendances and 110 outpatient appointments. The carbon footprint reduced by 3 tonnes through the fewer appointments in 2015 alone. One thousand and five hundred contacts were related to immunosuppression therapy monitoring. ${ }^{12}$ There were no reported breaches of clinical safety. The patient journey time to access specialist care at the time of a flare reduced from 6 weeks down to 1 week. Fifty active users of PKB in 2015 has since increased to 700 in 2018.

In the 2018 service evaluation, there was $100 \%$ response rate in both cohorts. In the prospective cohort, 17 of the 35 respondents were male and in the retrospective cohort, 13 of the 35 respondents were male. Two questions in the IBD-control questionnaire asks for the patient's own perception of IBD control. Sixty percent of prospective respondents reported that their IBD has been well controlled over the past two weeks when asked at the start of the study, increasing to $71 \%$ at 4 months. Eighty three percent of

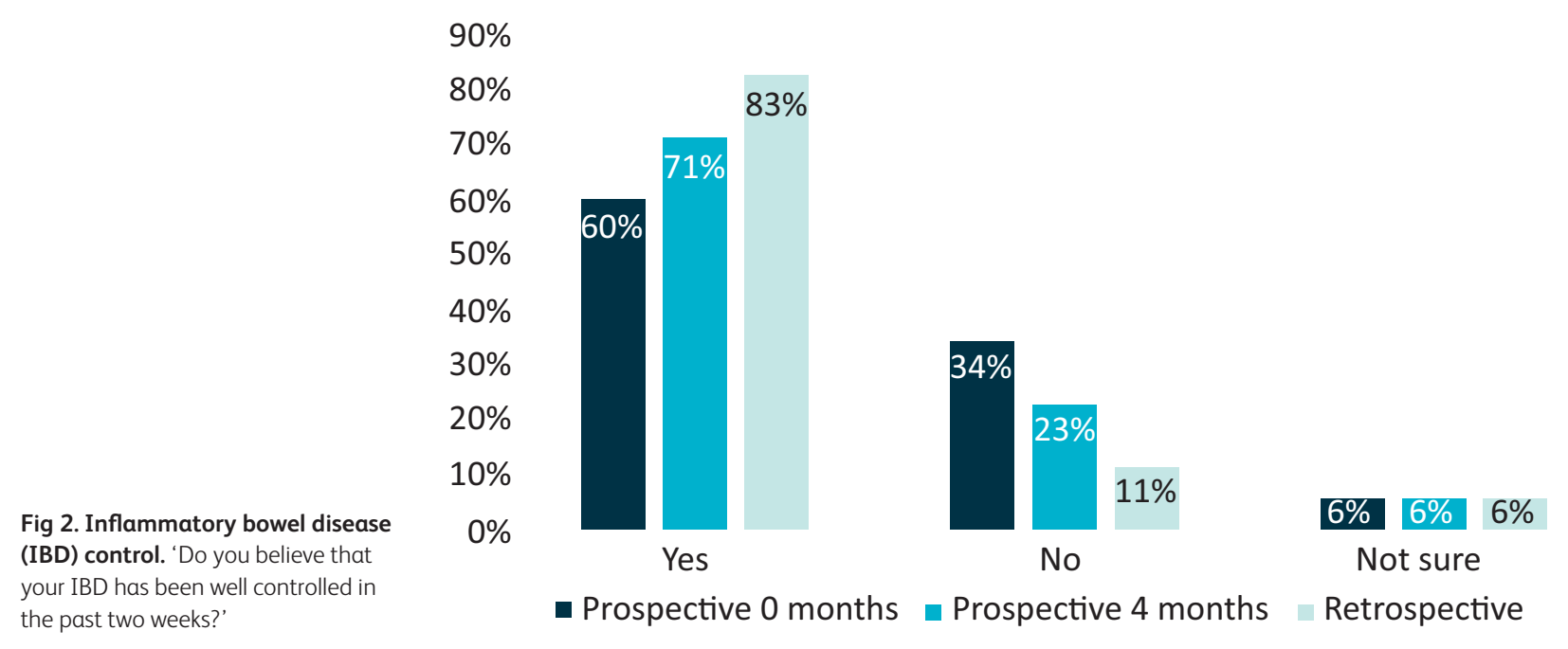


$45 \%$

$40 \%$

$35 \%$

$30 \%$

$25 \%$

$20 \%$

$15 \%$

$10 \%$

$5 \%$

$0 \%$

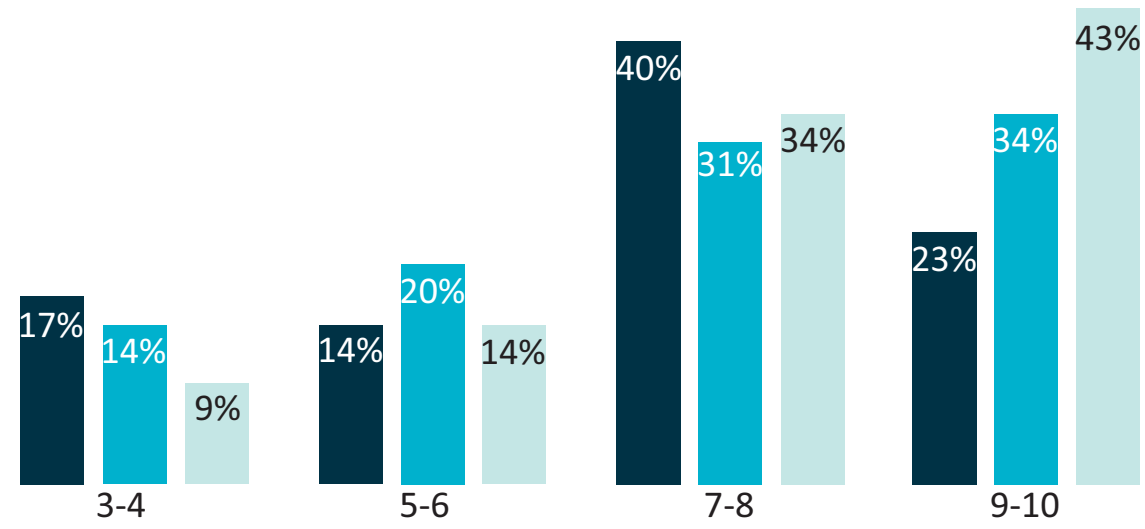

- Prospective 0 months

- Prospective 4 months

Retrospective

Fig 3. Inflammatory bowel disease (IBD) rating. 'How would you rate the overall control of your IBD in the past two weeks?' Linear scale from 1-10, 1 being worst possible control and 10 being best possible control.

retrospective respondents reported the same (Fig 2). Twenty three percent of prospective respondents rated their IBD control as 9 or 10 out of 10 over the past 2 weeks when asked at the start of the study, increasing to $34 \%$ at 4 months. Forty three percent of retrospective respondents reported the same (Figs 3 and 4 ).

Eighty nine percent of the prospective cohort at 0 months demonstrated low activation levels. This reduced to $63 \%$ at 4 months, with $37 \%$ demonstrating medium or high levels of activation, compared with $11 \%$ at baseline. Sixty six percent of the retrospective cohort demonstrated medium or high levels of activation (Fig 5).

Of the retrospective cohort, $68 \%$ said the NFTF service had a positive impact on their IBD, $77 \%$ said the NFTF service helped them feel more confident in managing their own health and $57 \%$ said the NFTF service had improved their quality of life (supplementary material S1).

Twenty of the 35 patients in the retrospective cohort left a free text comment, examples of which are include:

Helps me feel in control.

Someone there to answer questions when I need help. Made me more responsible in taking care of my health.
Easier to take control and contact professionals for advice when needed.

It has been a great comfort and reassurance. It helps me understand my illness and allows me to take control.

Eighteen of the 20 comments were positive. Of the two negative comments, one highlighted that the mobile app was not working and the other reported that messages were not responded to in a timely fashion.

\section{Discussion}

The new model of IBD care at ESH provides a locally generated approach to collaborative care with citizens being the assets driving the change. By redefining 'who does what where', it breaks down traditional boundaries between service user and provider through the provision of open access, so that patients may make informed decisions about their healthcare at a time and place convenient to them, a care model rarely seen in the NHS.

This intervention demonstrated an improvement in patient reported IBD control at 4 months. The retrospective cohort is used as a comparator as it is expected to demonstrate the long-term

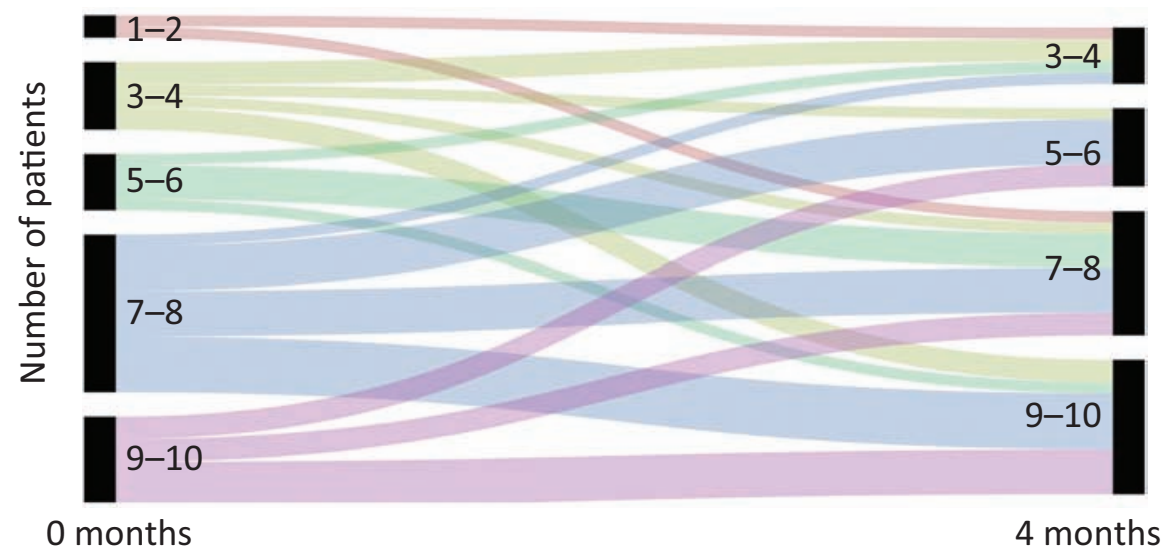

Fig 4. Alluvial diagram for overall inflammatory bowel disease (IBD) control in the prospective cohort from Fig 3. 


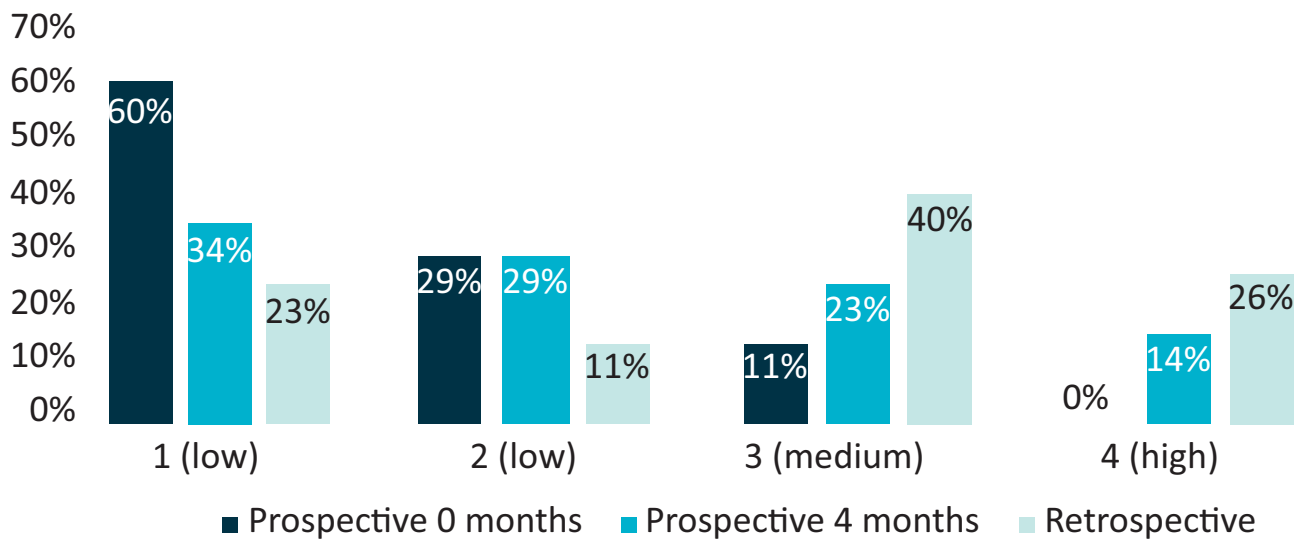

Fig 5. Patient Activation Measure ${ }^{\circledR}$ level (1, 2, 3 or 4).

impact of the intervention and a predictor for the ongoing impact on the prospective cohort beyond 4 months.

Despite better self-reported control of IBD in the prospective cohort at 4 months and in the retrospective cohort, up to $60 \%$ of people still reported symptoms, demonstrating that people can tolerate a degree of symptomatology associated with IBD if there is a perceived improvement of their condition over time. Less than $20 \%$ felt the need to change their medication (see supplementary material S1).

There is a right shift in the PAM ${ }^{\circledR}$ level scores in the prospective cohort at 4 months and in the retrospective cohort when compared with the prospective cohort baseline data. After controlling for demographics and health status, activated patients in America cost $\$ 1,987$ less per patient annually than lower activated patients. $^{16}$

The participants involved in the 2018 evaluation make up approximately $2 \%$ of ESH's IBD population. Although an improvement in $\mathrm{PAM}^{\circledR}$ and patient reported outcomes measures (PROMs) has been demonstrated, larger numbers need to be evaluated. To this effect, participant recruitment is ongoing aiming to evaluate data from a total of 100 patients in each of the prospective and retrospective cohorts, with additional data collection at 8 months in the prospective cohort. It is important to consider that recruits to the prospective cohort may have been recruited at a time of particular clinical need and would therefore be expected to demonstrate improvement. Longer-term data is required to understand better the impact of NFTF support on sustained improvement in PAM and PROMS.

The benefits of this model are realised on an individual, system and wider economical level. The patient engages in supported self-management, taking responsibility for their own health and uses clinical resource appropriately. The clinician with the greatest expertise is matched to the patient with the greatest clinical need, and this appropriate matching is seen at every clinical interaction. The NFTF structure provides timely advice and management not limited to opening hours, location or availability of staff. Medication can be used and escalated in an appropriate and timely way at scale. Information technology through the webbased portal provides the potential for spread across different specialties regionally and nationally.

A Danish group evaluated their web-guided approach to IBD care in 2010 and concluded it was feasible, safe and cost effective. ${ }^{17}$ Similar to our model of care, they provided open access with a web-based portal but required patients to complete an education programme. Moreover, they offered access only to those with mild-moderate disease severity. Our service does not require any prior training or education and is offered to all IBD patients regardless of severity, an important consideration in spreading this practice. A 2014 systematic review found that eHealth disease management was shown to generally improve quality of life, adherence, knowledge about the disease, and reduce healthcare costs in $\mathrm{IBD}^{18}$

In the UK, other IBD services are adopting similar web-based patient management portals and assessing their impact on patient experience. The IBD team in Salford have found that patients using their portal are more in control with greater independence to self-manage and have a better understanding of their condition, with a resulting improvement in patient experience. ${ }^{19}$ Similarly, over two-thirds of our retrospective cohort said the NFTF service had a positive impact on their IBD and helped them feel more confident in managing their own health and just over half said the NFTF service had improved their quality of life. Those who did not report an improvement in quality of life did not leave a free text comment and further detailed evaluation is required to understand the reasons behind their answers.

\section{Conclusion}

Our new model of care promotes citizens with long-term conditions as authors of their own health data and competent self-managers, enabling access to specialist support and clinical guidance appropriate to their situation through a technologydriven management solution. Citizens are supported in developing the confidence and skills to self-manage. With appropriate investigations and treatments initiated earlier and with continuous NFTF support provided, citizens take less time off work at times of flares and quality of life is improved. As their disease is better controlled, there is less risk of progressing to life-changing surgery. The majority of citizens value the autonomy of self-directed management and having the support of the specialist team at a time convenient to their individual situation.

We have demonstrated that management of long-term conditions can be delivered with fewer than the national recommended number of consultants and CNSs, delivering safe, effective, high quality care enhanced by technology, processes and 
pathways with the support of less costly up-skilled administrative staff.

The innovation is challenging current activity-based commissioning structures and aims to promote new commissioning models that focus on outcomes and support person-centred care.

Transformational change in any system is beyond the authority or ability of any one individual or team. Helping people see the wider system builds a shared understanding of complex or wicked problems and enables collaborating organisations to work together to develop solutions not evident to any individual part. ${ }^{20}$ Next steps should consider the widespread adoption of NFTF structures in IBD and other long-term conditions with multimethod prospective evaluation that includes patient activation, patient experience and clinical outcomes. This will provide the potential for group-based practice, bringing together patients that are dealing with similar issues to support each other and form a community of supported self-management.

\section{Supplementary material}

Additional supplementary material may be found in the online version of this article at http://futurehospital.rcpjournal.org:

$\mathrm{S} 1$ - Complete data set for prospective cohort at 0 months and 4 months, and retrospective cohort.

\section{References}

1 Department of Health. The NHS Constitution for England. DOH, 2015.

2 NHS England. Five Year Forward View. NHS, 2014.

3 Wilson T, Buck D, Ham C. Rising to the challenge: will the NHS support people with long term conditions? BMJ 2005;330:657.

4 Department of Health. Long term conditions compendium of information: third edition. DOH, 2012

5 Latella G, Papi C. Crucial steps in the natural history of inflammatory bowel disease. World J Gastroenterol 2012;18:3790.

6 Wagner EH. Chronic disease management: what will it take to improve care for chronic illness? Effective clinical practice 1998;1:2.

7 IBD Standards Group. Standards for the healthcare of people who have inflammatory bowel disease (IBD). IBD Standards Group, 2013.

8 Etchevers M], Aceituno M, Sans M. Are we giving azathioprine too late? The case for early immunomodulation in inflammatory bowel disease. World J Gastroenterol 2008;14:5512.
9 Eriksson C, Rundquist S, Cao Y, Montgomery S, Halfvarson J. Impact of thiopurines on the natural history and surgical outcome of ulcerative colitis: a cohort study. Gut 2018. https://gut.bmj.com/ content/early/2018/04/03/gutjnl-2017-315521.

10 Lu MJ, Qiu XY, Mao XQ, Li XT, Zhang HJ. Systematic review with meta-analysis: thiopurines decrease the risk of colorectal neoplasia in patients with inflammatory bowel disease. Aliment Pharmacol Ther 2018;47:318-31.

11 Colombel JF, Sandborn W], Reinisch W et al. Infliximab, azathioprine, or combination therapy for Crohn's disease. N Engl ] Med 2010;362:1383-95.

12 Reynolds R, Bull C, Street K et al. PTH-153 The Impact of an open access, non-face to face nurse led inflammatory bowel disease service on service transformation and patient outcomes. Gut 2016:65:A295.

13 Ghosh N, Premchand P. A UK cost of care model for inflammatory bowel disease. Frontline Gastroenterol 2015;6:169-74.

14 Hibbard JH, Greene J. What the evidence shows about patient activation: better health outcomes and care experiences; fewer data on costs. Health affairs 2013;32:207-14.

15 Bodger K, Ormerod C, Shackcloth D, Harrison M. Development and validation of a rapid, generic measure of disease control from the patient's perspective: the IBD-control questionnaire. Gut 2014;63:1092-102.

16 Greene J, Hibbard JH, Sacks R, Overton V, Parrotta CD. When patient activation levels change, health outcomes and costs change, too. Health Affairs 2015;34:431-7.

17 Elkjaer M, Shuhaibar M, Burisch ] et al. E-health empowers patients with ulcerative colitis: a randomised controlled trial of the web-guided 'Constant-care' approach. Gut 2010;59:1652-61.

18 Knowles SR, Mikocka-Walus A. Utilization and efficacy of internetbased eHealth technology in gastroenterology: a systematic review. Scand J Gastroenterol 2014;49:387-408.

19 Mclaughlin J, Stansfield SC, Lal S, Bodger K, Robinson JA. OTU-031 My-IBD portal: progress and impact update. Gut 2018;67:A223.

20 Senge $P$, Hamilton $\mathrm{H}$, Kania J. The dawn of system leadership. Stanford Social Innovation Review 2015;13:27-33.

Address for correspondence: Dr Antony Aziz, East Surrey Hospital, Redhill, Surrey RH1 1RH, UK.

Email: antony.aziz1@nhs.net 